\title{
Highly porous and interconnected starch-based scaffolds: Production, characterization and surface modification
}

\author{
Iva Pashkuleva *, Paula M. López-Pérez, Helena S. Azevedo, Rui L. Reis
}

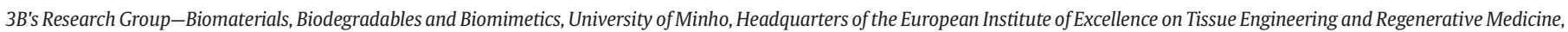
AvePark, Taipas, 4806-909 Guimarães, Portugal

IBB-Institute for Biotechnology and Bioengineering, PT Government Associated Laboratory, Braga, Portugal

\section{A R T I C L E I N F O}

\section{Article history:}

Received 13 January 2010

Received in revised form 30 March 2010

Accepted 28 April 2010

Available online 6 May 2010

\section{Keywords:}

Biomaterials

Processing

Starch

Surface modification

3D scaffolds

\begin{abstract}
A B S T R A C T
A convenient and straightforward process for preparation of highly porous and interconnected fiber mesh scaffolds with $50 \mathrm{wt} . \%$ content of starch is described. The proposed methodology avoids some of the previous encountered problems associated with the processing of starch-based materials such as thermal degradation, starch entrapment in the material bulk and inability to control/minimise the thickness of the fibers obtained by melt spinning, or low porosity and lack of interconnectivity for the scaffolds obtained by extrusion or injection moulding with blowing agent. Topographical characterisation of the obtained fibers revealed rough surface commonly related with increased cell attachment and growth. The in vitro tests with osteoblast cell line confirmed this trend and we observed higher cell number with increasing of the culture time. These results were also associated with protein adsorption from a complex solution where predominant adsorption of vitronectin over fibronectin was detected. Finally, a model modification by plasma was also carried out in order to confirm the versatility of these scaffolds by the possibility to further upgrade them via surface functionalisation. The in vitro tests confirmed that osteoblast-like cells proliferate faster on the modified scaffolds, which allows shortening the time needed for culturing prior to implantation.
\end{abstract}

(c) 2010 Elsevier B.V. All rights reserved.

\section{Introduction}

Starch is a natural polysaccharide, digestible by a whole host of living creatures ranging from bacteria to humans. This remarkable biodegradability distinguishes it from other abundant polysaccharides such as cellulose and makes it an excellent material for commercial exploitation. While textile, paper and packaging industries have acknowledged starch attractive properties and make it a significant part of their history [1-3], the biomaterials field started to exploit this polysaccharide only about 15 years ago [4,5]. The main problems associated with this delay are the poor mechanical properties of starch and its difficult processing; starch is a moisture sensitive polymer and pure starch products are very brittle [6,7]. Polymer blending is one of the strategies which allows overcoming these problems. In fact, starch-based blends have shown a great versatility being easily processed and used in different fields of application [3,8-10].

Starch blended with poly(ethylene-co-vinyl alcohol) (SEVA-C) is an excellent example confirming this versatility. Initially it was developed [11] and proposed as an alternative to the petrochemicals conventionally used in manufacturing plastic articles for single-use consumer

\footnotetext{
* Corresponding author. IBB-Institute for Biotechnology and Bioengineering, PT Government Associated Laboratory, Braga, Portugal. Tel.: + 351253 510907; fax: + 351 253510909.

E-mail address: pashkuleva@dep.uminho.pt (I. Pashkuleva).
}

application $[2,3,8,12]$ but in 1995 , Reis et al. [5] introduced it in the biomaterial field. The first studies on SEVA-C as a material for biomedical applications focused the mechanical properties [13,14], degradation behaviour [15-17] and biocompatibility [18,19] of compact samples produced by injection moulding. These studies have suggested that SEVA-C could be an excellent material for fabrication of devices for temporary orthopaedic applications. The proposed application imposed development of alternative processing methodologies which allow obtaining of three dimensional structures that resemble the morphology of bone and can successfully serve as a template for tissue ingrowth. Following this requirement, Gomes et al. have suggested the use of injection moulding [20] or extrusion with blowing agents at different ratios [21]. These techniques allowed control over the porosity but in both cases poor interconnectivity was reported for the obtained samples. To overcome this drawback, Pavlov et al. [22] have proposed fiber bonding scaffold fabrication. Polymer fibers exhibit an excellent surface area to volume ratio for enhanced cell attachment, making them a viable option as a scaffold material. Although high porosity and interconnectivity can be achieved by this technique, the described melt spinning of SEVA-C fibers does not permit precise control of the fiber diameter and the processing of thin fibers (the reported fiber diameter is $480 \mu \mathrm{m}$ ) is difficult. Moreover, previous studies have shown that starch is entrapped in a core-shell structure in the samples obtained from SEVA-C melt $[23,24]$; it is not present on the surface and therefore not readily accessible for bioentities recognition (e.g. enzyme degradation). Additionally, traces of low molecular weight polymer chains measured 
upon the immersion in an isotonic saline solution after short times evidenced some polymer degradation at the used processing temperature [17].

In this study, we have selected and described the wet spinning fabrication technique as an alternative method to obtain three dimensional SEVA-C scaffolds. This technique allows production of SEVA-C fibers from a solution instead of a melt. The processing involves spinning of a polymer solution with optimised viscosity/concentration into a coagulation bath where the scaffold is formed simultaneously at the bottom of this bath. The produced scaffolds were characterised in terms of morphology, surface properties, mechanical behaviour and cytotoxicity. We have also considered the possibility to further upgrade the obtained SEVA-C scaffolds by surface functionalisation. Hence, a model modification by plasma was carried out in order to confirm the versatility of the produced 3D structures.

\section{Materials and methods}

We have used a commercially available polymeric blend (trade mark name Mater Bi 1128RR, Novamont, Italy) of corn starch with 40/60 mol\% ethylene/vinyl alcohol copolymer (50/50 wt.\%, SEVA-C). The starch used to produce the blend has been obtained from native maize and its typical original composition is $70 \mathrm{wt} . \%$ amylopectin [ $\alpha(1 \rightarrow 4)$ - and $\alpha(1 \rightarrow 6)$-linked D-glucose] and $30 \mathrm{wt} \%$ amylose $[\alpha(1 \rightarrow 4)$-linked $D$-glucose]. All materials were supplied in granular form. Dimethyl sulfoxide (DMSO, Aldrich) was used without any further purification.

\subsection{Scaffolds production and surface modification}

Starch-based fiber mesh scaffolds were produced by wet spinning process schematically presented in Fig. 1. A polymer solution with a proper viscosity was obtained by dissolving of SEVA-C in dimethyl sulfoxide (DMSO) at a concentration of $5 \%(\mathrm{w} / \mathrm{v})$. This solution was loaded into a syringe and placed in a syringe pump (World Precision Instruments, UK). Each mesh was formed by extrusion of $1 \mathrm{~mL}$ SEVA-C solution into water coagulation bath. We have tested different speeds of extrusion and a speed of $2 \mathrm{~mL} / \mathrm{h}$ was found to be the most appropriate, resulting in formation of continuous and uniform fibers. The formed fiber bundles were washed several times with water in order to remove any residues of DMSO and then they were randomly displaced into a Teflon ${ }^{\mathrm{TM}}$ mould and dehydrated by ethanol with increasing concentration. The dried meshes were subjected to a thermal treatment $\left(160^{\circ} \mathrm{C}\right.$ for $30 \mathrm{~s}$ ) under a load of $0.15 \mathrm{~kg} / \mathrm{cm}^{2}$ to assure the bonding between neighbouring fibers. The final specimens were obtained by cutting of the fiber bonded meshes into circular shape with diameter of $1 \mathrm{~cm}$.
The surface treatment was performed using a plasma reactor PlasmaPrep $_{5}$ (Gala Instrument $\mathrm{GmbH}$, Germany) with a fully automated process control. Argon was used as a working gas and the pressure in the reactor was controlled ( 0.18 mbar) by adjusting its flow rate. Prior the treatment, the chamber was pursuit with argon five times. A radio frequency source $(13.56 \mathrm{MHz}$ ) was used and a power of $30 \mathrm{~W}$ was applied for $15 \mathrm{~min}$. The samples were kept $24 \mathrm{~h}$ after being removed from the reactor and then characterised and sterilised (ethylene oxide).

\subsection{Scaffolds morphology}

Scanning electron microscopy (SEM, LEICA Cambridge S360) and optical profilometry (Interferometric profiler Wyko-NT 1100, Veeco using Vertical Scanning Interferometry mode) were used to observe the structure of the formed scaffolds. Further details on the interconnectivity and porosity of the samples were obtained by microcomputed tomography $(\mu-\mathrm{CT})$ analyses. Three randomly selected samples from different batches were analysed with a high-resolution $\mu$-CT SkyScan 1072 scanner (Skyscan, Kontich, Belgium) using pixel size of $5.86 \mu \mathrm{m}$ and exposure time of $2.8 \mathrm{~s}$. The X-ray source was set at $30 \mathrm{kV}$ of energy and $180 \mathrm{~mA}$ of current. Approximately 400 projections were acquired over a rotation range of $180^{\circ}$ with a rotation step of $0.45^{\circ}$. Data sets were reconstructed using standardised cone-beam software (NRecon v1.4.3, SkyScan). Representative data sets of 90 slices were segmented into binary images with a dynamic threshold of 45 to 255 (grey values) and were used for morphometric analysis (CTAnalyser, v 1.5.1.5, SkyScan) and to build 3D models (ANT 3D creator, v2.4, SkyScan). The morphometric analysis included porosity, pore interconnectivity and mean pore size.

\subsection{Structural characterisation}

Fourier transform infrared spectroscopy (FTIR), thermo gravimetric analysis (TGA) and differential scanning calorimetry (DSC) were used to study the chemical composition of the blend before and after the processing and therefore to confirm the incorporation of all the blend components in the final scaffold. The FTIR spectra were recorded on a spectrophotometer IR-Prestige-21 (Shimadzu, Japan) with a resolution of $4 \mathrm{~cm}^{-1}$. While for the pure starch the spectrum was recorded in a transmittance mode using $\mathrm{KBr}$ tablets, an Attenuated Total Reflectance (ATR) device was employed to obtain the spectra of raw material and produced scaffolds. The results are presented as an average of 32 scans. The thermal degradation was measured using Elmer TGA7 equipment under a nitrogen atmosphere. The experiments were performed at a heating rate of $10^{\circ} \mathrm{C}$ in the temperature range of $50-600^{\circ} \mathrm{C}$. Differential scanning calorimetry (DSC) experiments were carried out in a Q100 calorimeter with controlled cooling accessory (TA Instruments, USA). The heat flow

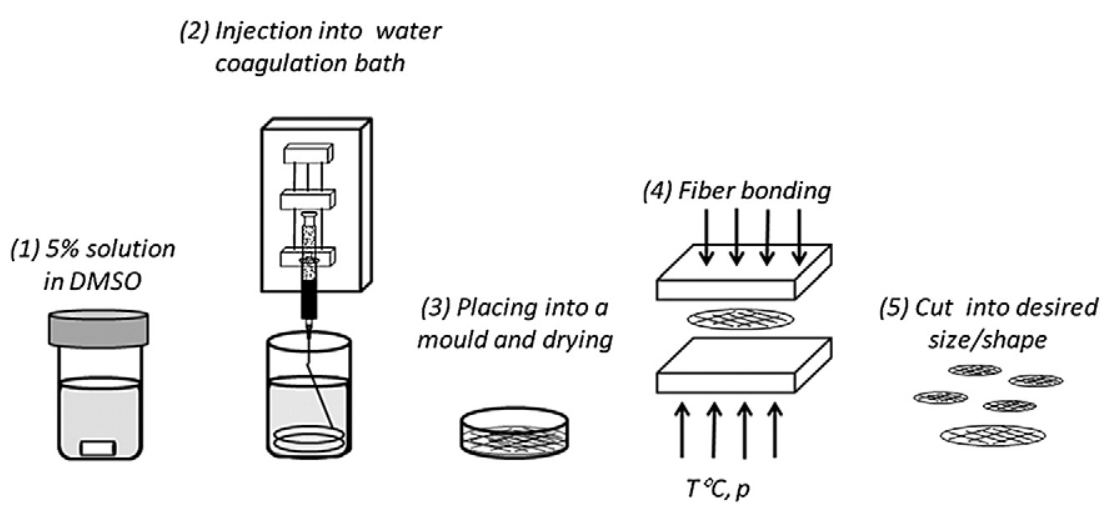

Fig. 1. Schematic presentation of the procedure used for SEVA-C scaffolds fabrication. 
calibration was performed with indium standard under the same conditions as the used for the samples analysis. All materials were sealed in Aluminium pans and the DSC scans were performed according to the following program:

(i) Initial temperature $20^{\circ} \mathrm{C}$;

(ii) First heating: heating rate of $10^{\circ} \mathrm{C} \mathrm{min}^{-1}$ to $230{ }^{\circ} \mathrm{C}$;

(iii) Cooling: cooling rate of $10^{\circ} \mathrm{C} \mathrm{min}^{-1}$ to $20^{\circ} \mathrm{C}$;

(iv) Second heating: heating rate of $10^{\circ} \mathrm{C} \mathrm{min}^{-1}$ to $230^{\circ} \mathrm{C}$.

X-ray diffraction (XRD) was used to examine changes in crystallinity as a result of the proposed processing methodology. The XRD patterns were collected between $2 \theta$ values from 6 to $60^{\circ}$, using a Bruker D8 Discover $\theta / 2 \theta$ diffractometer working with $\mathrm{Cu} K \alpha$ radiation.

\subsection{Mechanical properties}

Tensile tests (Universal Mechanical Testing Machine Instron 4505) were carried out at $25{ }^{\circ} \mathrm{C}$ and $50 \%$ of humidity. Tensile modulus, ultimate tensile strength and strain at break for single fibers were measured using a load cell of $2.5 \mathrm{~N}$ at crosshead speed $5 \mathrm{~mm} \mathrm{~min}^{-1}$. Three different sets of samples were tested: wet fibers, dry fibers and rewet fibers (dry specimens that were immersed in PBS for $5 \mathrm{~h}$ prior measurements). The tensile modulus was determined in the most linear region of the stress/strain curve using the secant method. The data are presented as an average \pm standard deviation.

The storage $\left(E^{\prime}\right)$ and the loss modulus $\left(E^{\prime \prime}\right)$ for the scaffolds were measured using dynamic mechanical analysis (DMA). E' (the elastic component) is related with the stiffness of the material, while $E^{\prime \prime}$ (the viscous component) is associated with the dissipation of energy due to internal friction at the molecular level. The loss factor, $\tan \delta$, is defined as the ratio $E^{\prime \prime} / E^{\prime}$. The viscoelastic measurements were performed using a TRITEC8000B DMA from Triton Technology (UK), equipped with the compressive mode. The measurements were carried out at room temperature and samples with circular shape $(d=1 \mathrm{~cm})$ were used. The wet, dry and rewet samples were clamped in the DMA apparatus and the measurements were performed in the range of $0.1-50 \mathrm{~Hz}$ of frequency. The experiments were performed under constant strain amplitude $(30 \mu \mathrm{m})$.

\subsection{Water uptake}

The hydration degree of the fibers was studied and compared with this one for the compact samples. Hence, compact samples and scaffolds were immersed in a phosphate buffered saline (PBS) solution $(0.01 \mathrm{M}, \mathrm{pH} 7.4)$ at $37^{\circ} \mathrm{C}$. At the end of each predetermined period, the samples were removed from the solution, washed, gently blotted with a paper to remove the excess of the liquid and weighted. The percentage of water uptake was calculated as:

\%water uptake $=\left[\left(M_{\text {end }}-M_{\text {initial }}\right) / M_{\text {initial }}\right] \times 100$

where $M_{\text {initial }}$ is the initial weight of the sample and $M_{\text {end }}$ is the weight of the sample after removing from the solution. Triplicate samples were used for each condition. The average water uptake values \pm standard deviation are plotted as a function of time.

\subsection{Surface properties}

Cells interact with scaffolds primarily through the material surface which can be dominated by the surface chemical and topological features. X-Ray photoelectron spectroscopy (XPS) was used to characterise the surface chemistry of treated and untreated samples. The analysis was carried out using an instrument VG Escalab 250 iXL (VG Scientific, UK). Charge compensation at the surface was performed using both a low energy flood gun (electrons in the range of 0 to $14 \mathrm{eV}$ ) and an electrically grounded stain steel screen placed directly onto the sample surface. The measurements were carried out using monochromatic Al$\mathrm{K} \alpha$ radiation $(\mathrm{h} \nu=1486.6 \mathrm{eV})$ in a Constant Analyzer Energy mode (CAE) with $100 \mathrm{eV}$ pass energy for survey spectra and $20 \mathrm{eV}$ pass energy for high-resolution spectra. Photoelectrons were collected from a take-off angle of $90^{\circ}$ relative to the sample surface. Charge referencing was adjusted by setting the lower binding energy C1s hydrocarbon $\left(\mathrm{CH}_{x}\right)$ peak at $285.0 \mathrm{eV}$. Surface elemental composition was determined by standard Scofield photoemission cross sections. The chemical functional groups identity was obtained from the high-resolution peak analysis of carbon-1s (C1s), envelopes by XPSPEAK 4.1 software.

The surface topology was studied by optical profilometry. This non-contact method allows roughness analysis of 3D structures with high resolution from subnanometer to millimetre step-height. In this study an optical profiler WykoNT3300 from Veeco Instruments Inc. was used. The measurements were performed in phase shifting interferometry (PSI) mode and an area of $2 \mathrm{~mm}$ was analysed with a vertical resolution of $3 \mathrm{~nm}$.

\subsection{In vitro characterisation}

The protein system used in this study was a multi-protein solution of $10 \mathrm{v} / \mathrm{v} \%$ heat-inactivated fetal bovine serum (FBS; Biochrom AG, Germany) in Dulbecco's modified Eagle's medium (DMEM; SigmaAldrich, Inc, USA). Unmodified and plasma treated SEVA-C scaffolds were incubated for $1 \mathrm{~h}$ at $37^{\circ} \mathrm{C}$ in this solution. Samples immersed in Phosphate Buffered Saline solution (PBS; Sigma-Aldrich, Inc, USA), were used as blanks. Two adhesion proteins were studied: fibronectin (Fn) because it is commonly used in a standard procedure applied to improve adhesion of cells and Vitronectin ( $\mathrm{Vn}$ ) because of its influence on cell spreading and migration. After the incubation time was over, the samples were fixed in a 2.5\% formalin solution for $5 \mathrm{~min}$, washed with PBS and incubated at room temperature for $1 \mathrm{~h}$ with primary antibody mouse anti-cow Vn (Santa Cruz, USA) or mouse anti-cow Fn (Santa Cruz, USA). Both primary antibodies were diluted at ratio 1:50 (v/v) in 1\% (w/v) Bovine Serum Albumin (BSA, SigmaAldrich, USA) solution in PBS. All samples were again washed and incubated for $1 \mathrm{~h}$ at room temperature with goat anti-mouse Alexa Fluor $488 \operatorname{IgG}(\mathrm{H}+\mathrm{L})$ secondary antibody (Invitrogen, USA). Labelled samples were analysed by an Olympus IX81 Confocal Laser Scanning Microscope (CLSM).

Cell attachment and proliferation onto the scaffolds were tested by an osteosarcoma cell line (SaOs-2), an immortalized cell line with an osteoblastic phenotype, which was obtained from European Collection of Cell Cultures (ECACC, UK). The cells were cultured in DMEM supplemented with $10,000 \mathrm{U} / \mathrm{mL}$ penicillin-G sodium, $10,000 \mu \mathrm{g} / \mathrm{mL}$ streptomycin sulfate and $25 \mu \mathrm{g} / \mathrm{mL}$ amphotericin B in a $0.85 \%$ saline (Gibco, Invitrogen Corporation, UK) and $10 \%$ of FBS in a humidified atmosphere with $5 \%$ of $\mathrm{CO}_{2}$. A suspension of $3 \times 10^{6}$ cells was added to each scaffold. The scaffolds were incubated for 1,3 and 7 days under standard culture conditions $\left(37^{\circ} \mathrm{C}, 5 \% \mathrm{CO}_{2}\right.$, humidified atmosphere). The morphology of SaOs-2 cells was observed by Scanning Electron Microscopy (S360, Leica Cambridge, UK). Cells were fixed using 2.5\% glutaraldehyde (Sigma, USA) solution in PBS. Prior the analysis, the samples were dehydrated by graded ethanol solutions.

DNA quantification was used to evaluate cell proliferation. Cells were lysed by osmotic and thermal shock and the obtained supernatant was used for DNA analysis. DNA content along the time of culture was determined using the PicoGreen dsDNA kit (MolecularProbes) and the fluorescence was read $(485 \mathrm{~nm} / 528 \mathrm{~nm}$ of excitation/emission) in a microplate reader. The DNA amounts were calculated from a standard curve. Triplicates were analysed at each time point. All the data were subjected to statistical analysis and are reported as mean \pm standard deviation. The normality of the data was checked by applying the Shapiro-Wilk's $W$-test. Because all the samples follow a normal distribution, Student $t$-tests for independent 


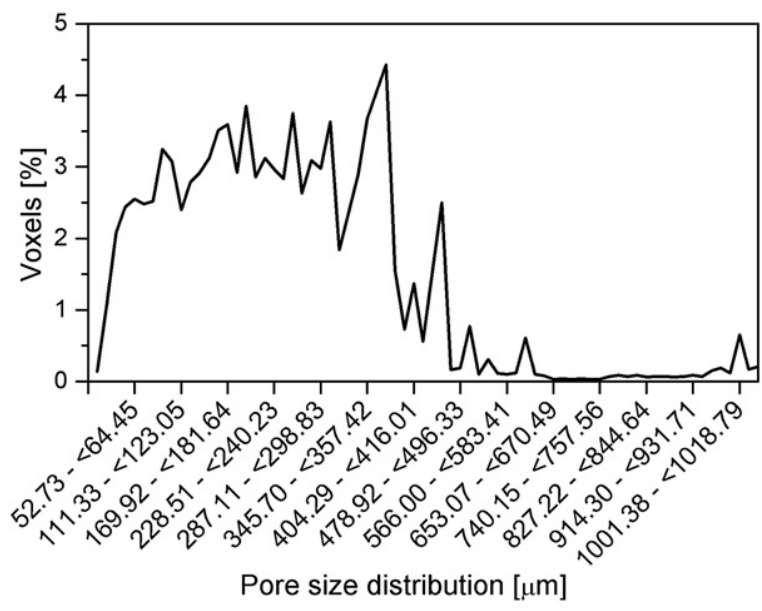

Fig. 2. Pore size distribution for the produced scaffolds.

samples were performed. The differences were considered statistically significant if $p<0.05$.

Cell viability was assessed by staining with the fluorochrome dye calcein-AM (MolecularProbes). The samples were treated with $0.002 \%$ calcein-AM solution in DMEM culture medium and incubated at dark for $15 \mathrm{~min}$ at $37^{\circ} \mathrm{C}$ in a humidified atmosphere of $5 \% \mathrm{CO}_{2}$. Cell fluorescence was examined by an Axioplan Imager Z1 from Zeiss, Germany.

\section{Results and discussion}

Three dimensional (3D) scaffolds serve as temporary substrates for supporting and guiding tissue formation in various in vitro and in vivo tissue regeneration strategies. In their design much effort has been focused on optimisation of parameters such as pore size, porosity and interconnectivity because they determine the mass transport in the scaffold, i.e. how fluids, solutes and cells move in and out of the construct. In bone engineering, there is a general consensus that pores bigger than $100 \mu \mathrm{m}$ may favour higher alkaline phosphatase activity and more bone formation although the optimal pore size varies with the scaffold material and other parameters (e.g. tortuosity) [25]. Moreover, Kuboki et al. [26] demonstrated that in hydroxyapatite scaffolds loaded with bone morphogenic proteins pores in the range of $90-120 \mu \mathrm{m}$ induced cartilage formation fol- lowed by bone formation while larger diameter of $350 \mu \mathrm{m}$ induced bone formation directly and the reason for this result is the difference in the onset time of vascularization and cell differentiation. We have used $\mu$-CT to determine the size of the pores in the wet spun SEVA-C scaffolds. Fig. 2 represents the distribution of the porous size in one of these scaffolds. As can be seen from the graph, the sample presented wide pore size distribution in the range of 60-500 $\mu \mathrm{m}$. Similar data were obtained for the other tested samples. However, when we averaged the mean pore size for the three studied specimens, we have obtained a value of $236.5 \pm 11 \mu \mathrm{m}$. This small standard deviation demonstrates that the procedure proposed herein is reproducible and it can generate scaffolds with similar porosity.

Other parameters which influence the transport within the scaffold are porosity and interconnectivity. While the need of high interconnectivity is obvious, not always very high porosity is desired since it could result in diminishing the surface area available for cell attachment in 3D scaffold. Fig. 3 shows the size, shape and morphology of the scaffolds obtained by wet spinning. The images represent randomly distributed fibers with diameter about $100 \mu \mathrm{m}$ which are forming highly interconnected and porous scaffolds. The mCT analysis confirmed this observation and demonstrated that reproducible $3 \mathrm{D}$ structures with $80.5 \pm 0.1 \%$ mean porosity and interconnectivity of about $97 \%$ can be obtained at the used conditions.

Because cells interact with the scaffolds primarily through the material surface, the surface chemistry and topological features are of central importance in directing cell-material interactions. Generally, surface roughness increase is associated with an enhanced cell adhesion and migration and with higher production of extracellular matrix (ECM). However, this response is dimension-dependent; whereas micro-features increased the attachment and growth, the nanoscale roughness induces enhanced cell differentiation [25]. Due to both phase inversion during the formation of the fibers and the applied drying process, the produced fibers presented non-smooth surface as it can be seen in the optical profiler micrographs (Fig. 3D) and therefore, they differ from the fibers obtained by melt spinning [22]. Values of $559.31 \pm 40.4 \mathrm{~nm}$ for roughness average ( $\mathrm{Ra}$ ) and $741.00 \pm$ $23.8 \mathrm{~nm}$ for root mean square $(\mathrm{Rq})$ were obtained by optical profilometry analysis of the roughness of the fibers. Different processing techniques can also evoke changes in the surface chemistry. We have analysed the surface elemental composition by XPS but we did not find a significant difference between the values obtained for wet spun fibers ( $\mathrm{C}: \mathrm{O}$ ratio of $1: 0.27$ ) and the one previously reported for injection moulded compact samples ( $\mathrm{C}: \mathrm{O}=1: 0.31[23])$.
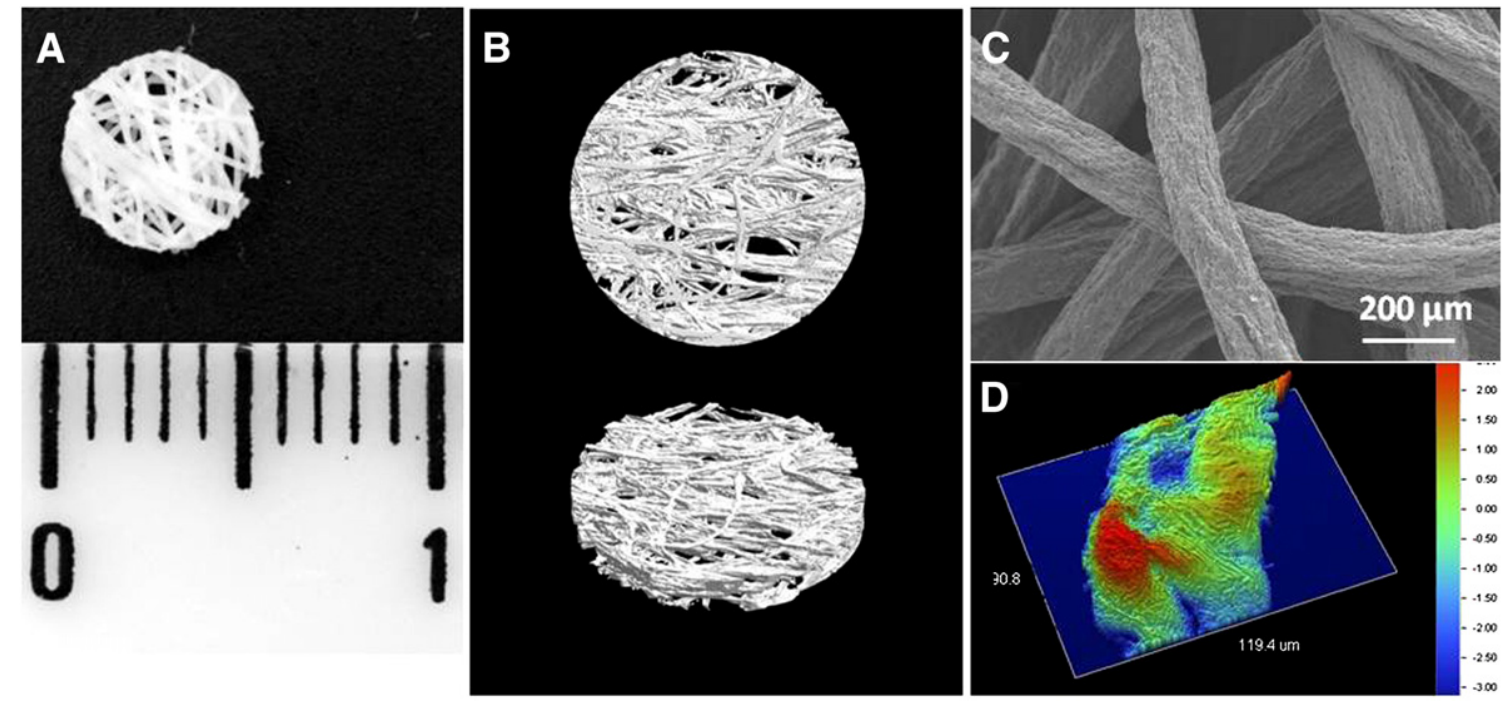

Fig. 3. SEVA-C scaffolds produced by wet spinning. Images were obtained by digital photography (A); $\mu-C T$ (B); SEM (C) and optical profilometry (D). 


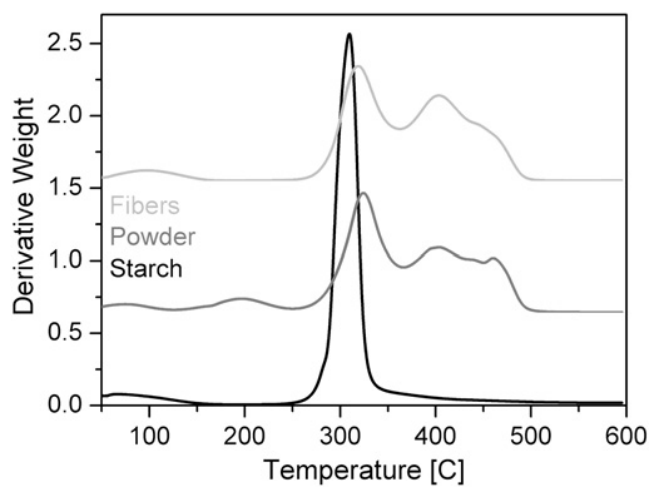

Fig. 4. TGA thermograms of starch, raw blended material and the obtained wet spun fibers.

Possible changes of the bulk composition of SEVA-C upon the processing caused by degradation or dissolving of some of the blend components in the precipitation bath were also investigated (Figs. 4 and 5). Three weight loss processes can be observed in the TGA spectra (Fig. 4) of both raw material and processed scaffolds: the peak at about $70{ }^{\circ} \mathrm{C}$ corresponds to water desorption; loss of plasticiser (glycerol) occurs at about $200{ }^{\circ} \mathrm{C}$ and a complex degradation process in the range of $300-550^{\circ} \mathrm{C}$. A comparison with the spectrum of native starch demonstrated that the first part of this complex signal with a maximum at about $300{ }^{\circ} \mathrm{C}$ is consistent with the degradation of starch and therefore confirms its presence in the produced scaffolds.

The FTIR-ATR spectra confirmed this result. The spectrum of pure starch is presented on Fig. 5A where the characteristic bands corresponding to $-\mathrm{OH}$ groups $\left(3450 \mathrm{~cm}^{-1}\right)$, glycosidic $-\mathrm{O}-\mathrm{C}-\mathrm{O}-$ bonds (1150-1040 $\mathrm{cm}^{-1}$ ) and the $-\mathrm{CH},-\mathrm{OH}$ vibrations from the glucose ring are shown. The presence of glycosidic band in the spectra of the used raw material and the scaffolds produced from it (Fig. 5B) corroborates
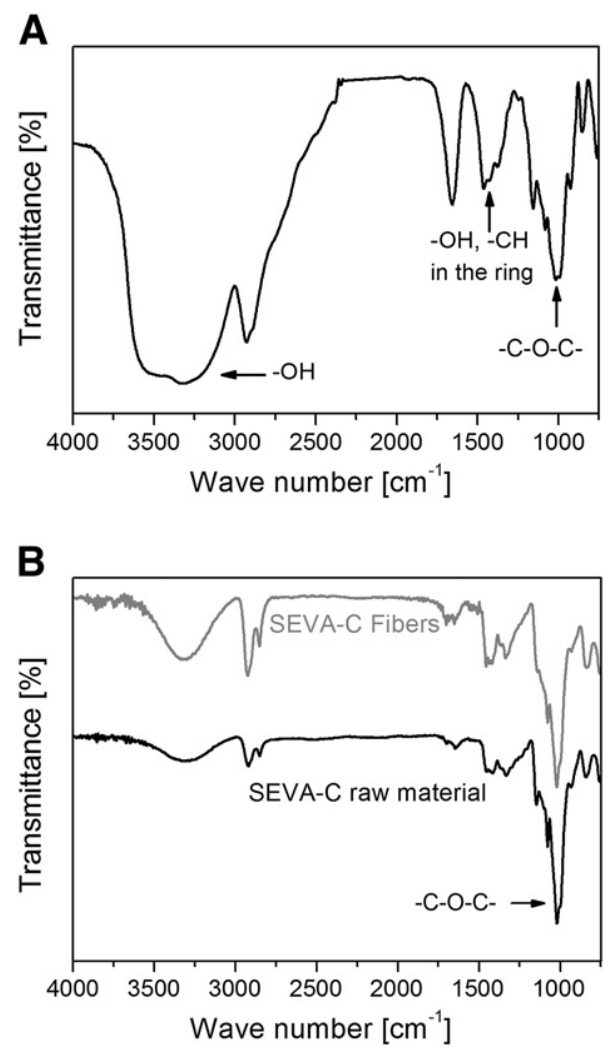

Fig. 5. FTIR spectra of pure starch (A), SEVA-C raw material and the processed SEVA-C fiber meshes (B). the successful incorporation of starch in the blend and in the final constructs. Moreover, no change in the spectrum (specifically in the $1150-1040 \mathrm{~cm}^{-1}$ band) of SEVA-C after processing was detected. This result demonstrates that the used methodology does not induce any hydrolytic degradation of starch.

Further structural characterisation was carried by DSC and XRD. As it was expected, the DSC thermograms (Fig. 6) did not show any transitions arising from starch. The broad signal observed at the first heating scan is related with disruption of hydrogen bonds and with water release.

This hypothesis is also in accordance with TGA results which suggested loss of water at temperature above $50{ }^{\circ} \mathrm{C}$ and with the thermograms obtained at the second heating scan where this peak does not appear. The most pronounced endothermic peak with an onset at $162{ }^{\circ} \mathrm{C}$ was assigned to the melting of the poly(ethylene-covinyl alcohol) fraction and its enthalpy was found to be $13.7 \mathrm{~J} / \mathrm{g}$ for the wet spun fibers and $20.76 \mathrm{~J} / \mathrm{g}$ for the scaffolds produced from them. Similar difference has been observed before for the blend starch/poly (ethylene-co-vinyl alcohol) and it has been reported $[27,28]$ that the enthalpy of this transition depends strongly on the moisture content and could change significantly with its variation.

Besides the material chemistry, different processing approaches primarily focused on changes of mechanical properties of the produced specimens. Hence, we expected that the wet spun fibers will present properties that differ significantly from those reported for fibers produced by melt spinning. Since biomedical application is intended, the behaviour of the produced structures at physiological conditions is of main importance. Therefore, the mechanical tests were performed for wet (as produced, before drying) fibers, for dry samples and for samples immersed in PBS for $5 \mathrm{~h}$ prior the measurements. The results (Table 1 ) demonstrated that the obtained dry fibers are stiffer when compared to the melt spun SEVA-C fibers (84 MPa [22]). This result can be explained with the applied drying procedure; the samples were dried by ethanol with increasing concentration that extracts all the water (plasticising effect) included in the fibers. Both Esec and UTS values are lower than the reported for the melt spun fibers. However, different strategies can be applied to improve the mechanical properties of the obtained fibers. In fact, it has been shown for melt spun SEVA-C fibers that their modulus can be increased 7 times by stretching them (additional orientation) at the end of the processing [22].

The mechanical properties of the scaffold arise from a combination of the properties of the bulk polymer, the geometry of the scaffold and the fabrication technique. Initially, we have tested how the sintering process applied at the end of the methodology proposed by us changes the crystallinity of the obtained scaffolds. Although applied for very short time, the sintering process altered significantly the crystallinity of the produced scaffolds as can be seen from the diffraction patterns presented in Fig. 7; greater intensity and full width at half maximum (FWHM) values at $2 \theta=20^{\circ}$ were measured for the final scaffolds than for the wet spun fibers.

The isothermal DMA response of these scaffolds as a function of frequency in terms of storage modulus $\left(E^{\prime}\right)$, and loss factor $(\tan \delta)$ is shown on Fig. 8A.

The obtained modulus and strength values are much lower than that of cortical bone which typically has a modulus value of 7-30 GPa and strength of $50-150 \mathrm{MPa}$ [29]. This is expected since the bone is a composite material; the presence of hydroxyapatite in its structure contributes significantly for these high values. Although the scaffolds fabricated and tested in this study are not intended for load sharing applications, their mechanical properties can be further improved by adding ceramic fillers.

As it was expected, the modulus of the wet scaffolds decreases significantly due to the adsorbed water (Fig. 8B) and its plasticisation effect. The adsorbed water causes higher damping (the material loose more deformation energy) and this is reflected in the higher values of 

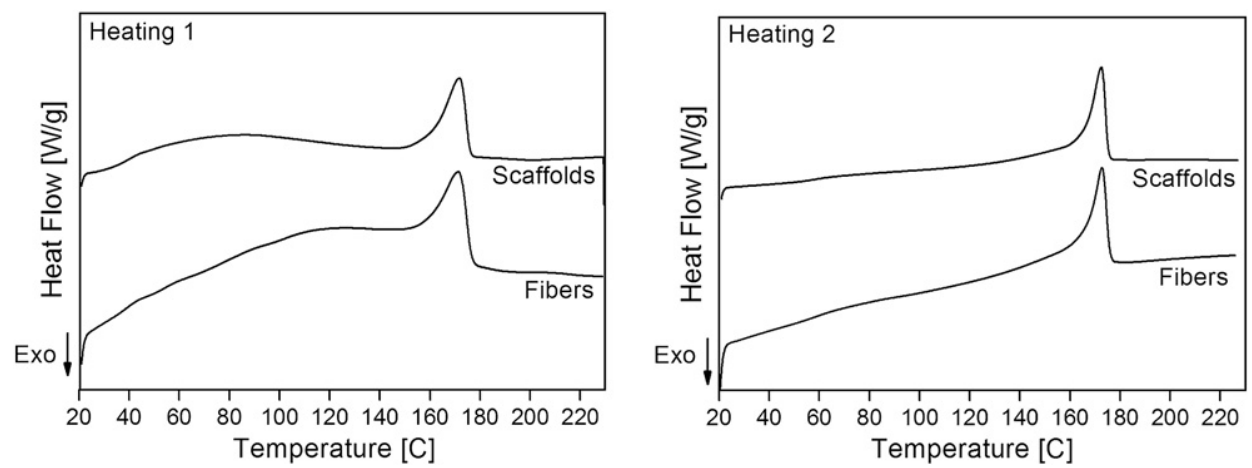

Fig. 6. DSC curves for the first and second heating of the fibers and the scaffolds porduced from them.

Table 1

Tensile modulus $\left(E_{\mathrm{sec}}\right)$, ultimate tensile strength (UTS) and strain at break for the produced fibers in dry and wet state.

\begin{tabular}{lccc}
\hline Sample & $E_{\text {sec }}[\mathrm{MPa}]$ & UTS $[\mathrm{MPa}]$ & Strain at break $[\%]$ \\
\hline Wet fibers & $1.2 \pm 0.4$ & $0.3 \pm 0.1$ & $93.3 \pm 9.7$ \\
Dry fibers & $388.2 \pm 88.0$ & $9.0 \pm 1.0$ & $5.1 \pm 1.4$ \\
Re-wet fibers & $1.9 \pm 0.4$ & $0.3 \pm 0.1$ & $47.4 \pm 4.5$ \\
\hline
\end{tabular}

loss factor obtained for the wet scaffolds. It can be also seen that the loss factor increases with frequency; shorter time between each cycle is not enough for the macromolecules to deform and therefore to evoke lost of energy.

Patient safety is the paramount concern for any tissue engineering application. The bulk material and its degradation products must be biocompatible and clearable by the body. It has been demonstrated by several in vitro and in vivo studies that SEVA-C is a non-cytotoxic material [30-32]. However, the chosen processing technique can affect the biocompatibility of the material since the generated surface features (chemistry and topography) can be quite different. Fig. 9 shows SaOs-2 seeded onto SEVA-C scaffolds after 1 and 7 days of culture.

As it can be seen cells do adhere to the surface of the produced scaffolds. The morphology and the shape of the cells were different along the culture time. Whereas at the initial period some of the SaOs2 presented a round shape, after 7 days of culture all cells were spread with extended filopodia making bridges between the fibers. CalceinAM assay confirmed that cells remained viable through all the studied period and DNA quantification evidenced their proliferation along the cultured time.

Previous studies have shown that plasma treatment can additionally improve cell attachment and spreading onto compact samples produced by injection moulding [33]. Plasma surface modification is a

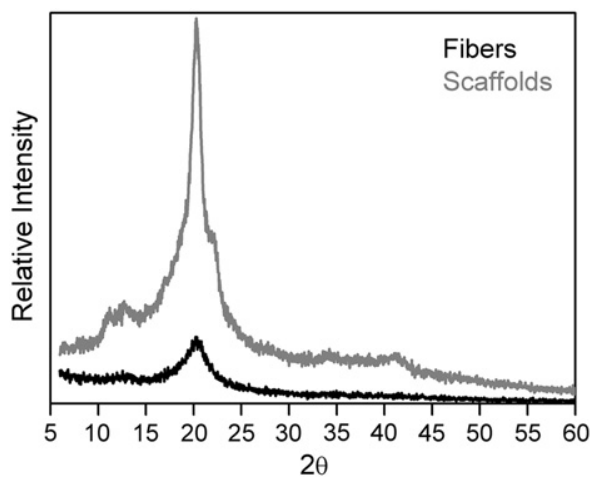

Fig. 7. X-ray diffraction patterns, normalized to the same total intensity, for the wet spun fibers and the scaffolds produced from them by sintering. very convenient technique for tailoring material surface functionality and topography without altering material bulk properties (e.g. biodegradability, mechanical properties, etc.). Recently, we have demonstrated [34] that plasma modification can be also applied to 3D scaffolds when they possess enough high porosity and interconnectivity. In this study, we have used Ar plasma modification as a model surface treatment. The proposed treatment can be further adapted to plasma grafting of different monomers.

Surface characterisation revealed a slight increase in the surface roughness as a result of the etching processes usually observed during the plasma treatment. The obtained values were $\mathrm{Ra}=672.85 \pm 19.9 \mathrm{~nm}$ and $\mathrm{Rq}=858.50 \pm 91.6 \mathrm{~nm}$, respectively. A significant difference was observed for the surface chemical composition (Fig. 10). The XPS spectra showed that the oxygen content increases as a result of the treatment
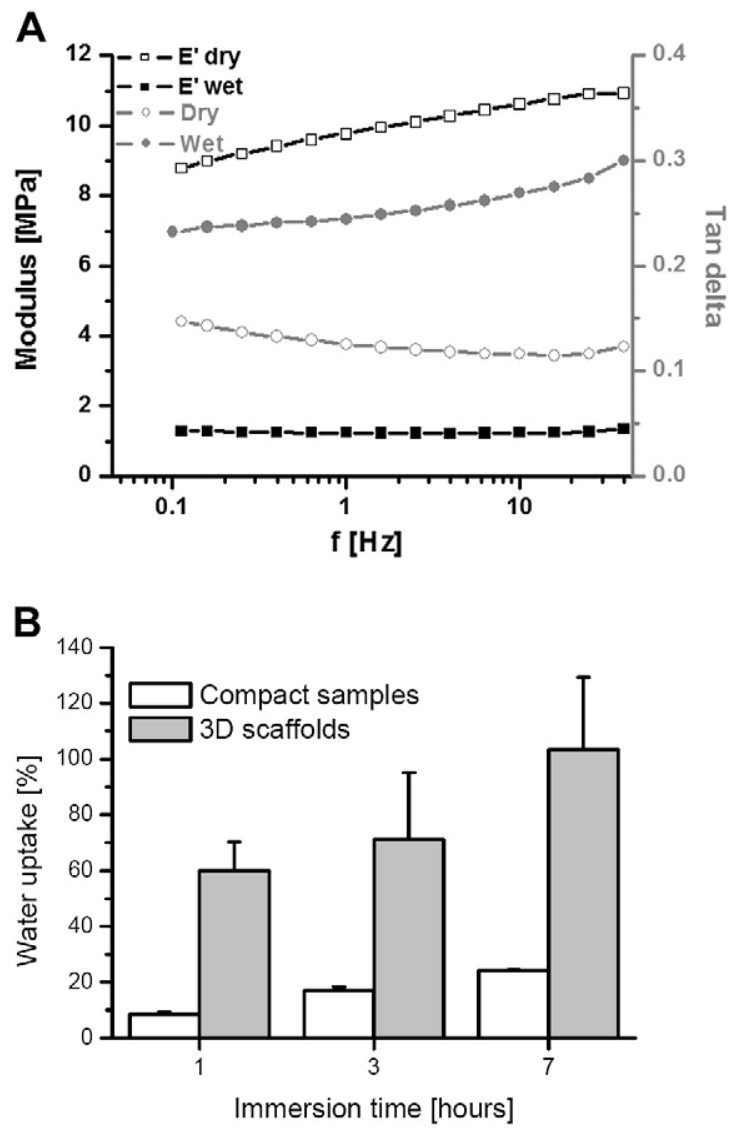

Fig. 8. Storage modulus $\left(E^{\prime}\right)$ and loss factor $(\tan \delta$ ) versus log frequency obtained at room temperature (A) and water uptake ability (B) of injection moulded compact samples and 3D scaffolds immersed in isotonic saline solution $(0.154 \mathrm{M} \mathrm{NaCl}, \mathrm{pH}=7.4$, room temperature). 

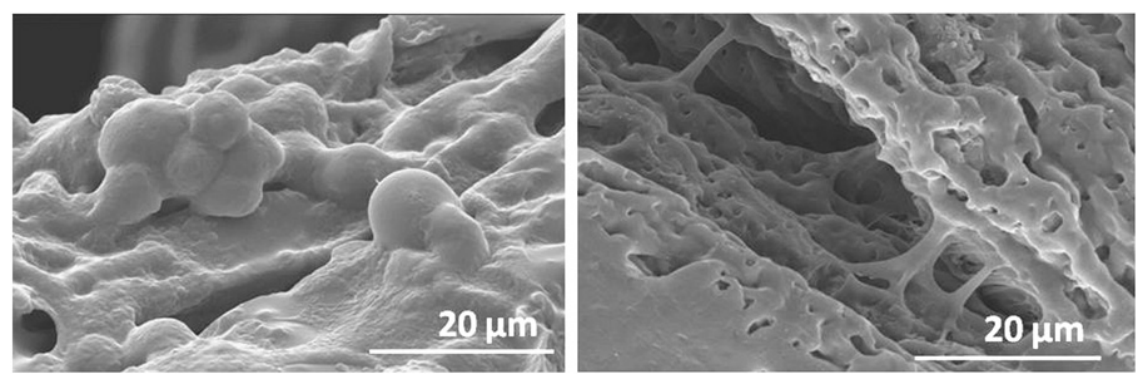

Fig. 9. SEM micrographs of SaOs-2 cultured on SEVA-C scaffolds for 1 (left) and 7 (right) days.
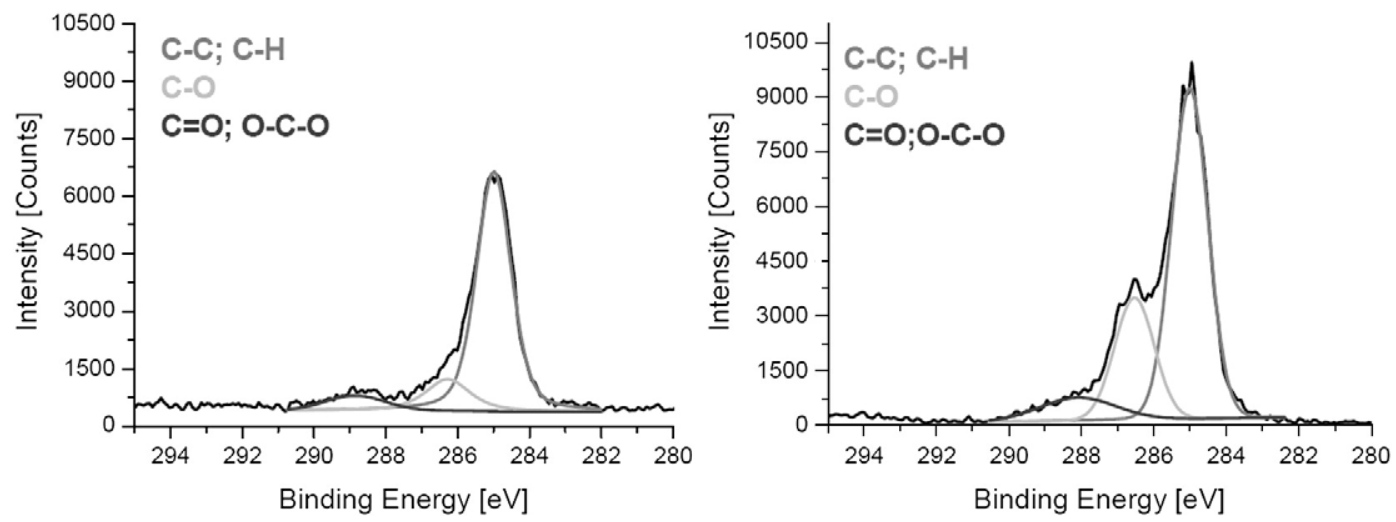

Fig. 10. C1s core level spectra of SEVA-C before (left) and after the applied treatment.
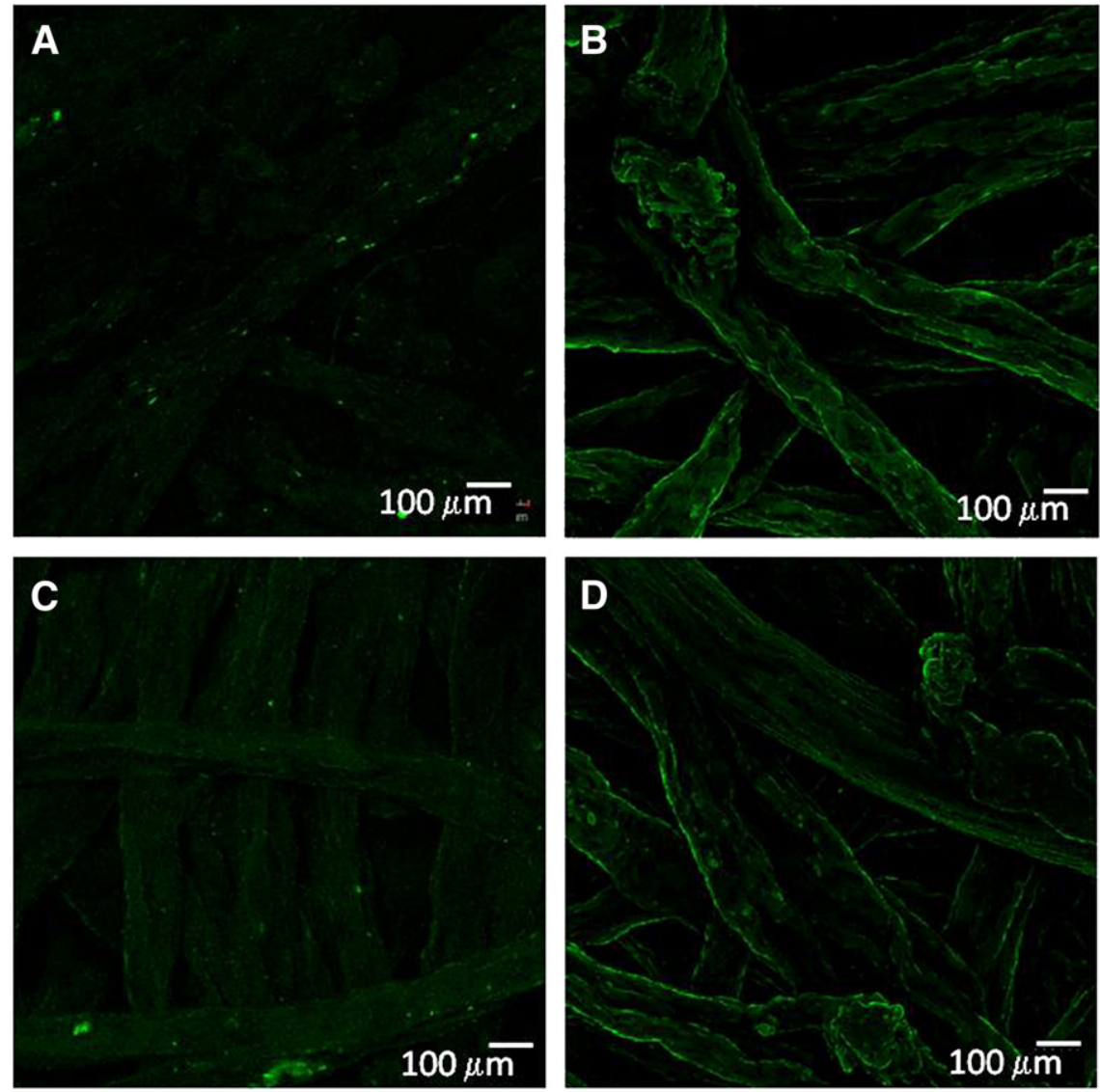

Fig. 11. Untreated (A, B) and plasma-modified (C, D) SEVA-C fiber meshes, immunolabeled for Fn (A, C) and Vn (B, D) after 1 h immersion in culture medium. 

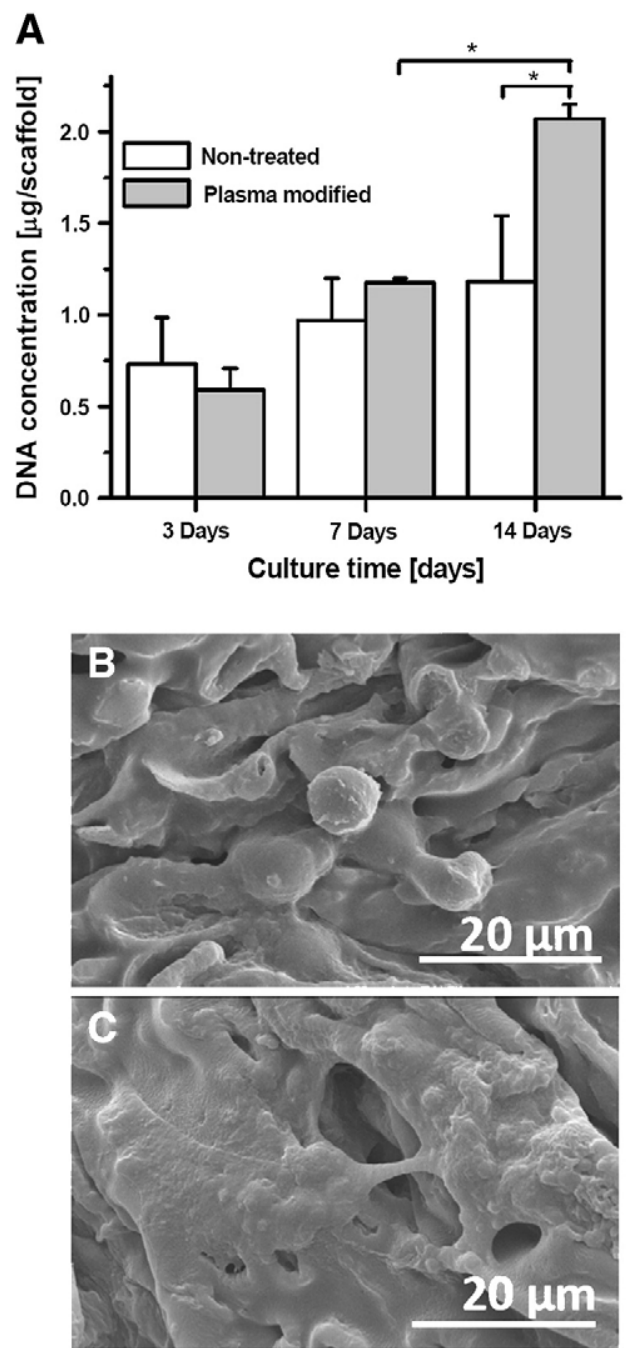

Fig. 12. Cell proliferation (A) and morphology (B, C) of SaOs-2 grown on SEVA-C scaffolds. SEM micrographs were taken for cells cultured for 1 day on untreated (B) and modified (C) fiber meshes. *Significantly different $(t$-test, $p<0.05)$. and the $\mathrm{C}: \mathrm{O}$ ratio changes from 1:0.27 for untreated SEVA-C scaffolds to 1:0.74 for modified ones. The C1s core level spectra demonstrated that this increase is mainly due to the $\mathrm{C}-\mathrm{O}(\mathrm{H})$ bonds, which means that oxidation takes place on the surface as a result of the plasma treatment.

Because cells are extremely sensitive to their environment, the introduced changes in the surface chemistry and topography can alternate their behaviour. It is well described that in the presence of a foreign material, proteins from blood and/or from interstitial fluids will cover the material surface almost instantaneously and it is this protein layer which will translate the surface structure and composition into a biological language. We have studied the adsorption of two adhesive proteins: fibronectin ( $\mathrm{Fn}$ ) was chosen because it is the best studied model adhesive protein. On the other hand, previously published studies carried out with SEVA-C compact samples reported that from complex protein solutions the amount of adsorbed vitronectin $(\mathrm{Vn})$ is higher than that of Fn [35]. However, the analysis of the adsorption of these biomolecules on 3D SEVA-C scaffolds has not been demonstrated.

As for the compact samples, the micrographs presented on Fig. 11 show markedly increased adsorption of Vn compared to Fn. Although higher $-\mathrm{OH}$ surface concentration obtained after the plasma treatment was expected to result in enhanced $\mathrm{Vn}$ adsorption [36], the observed homogeneous protein distribution was similar for both Vn-labelled untreated (Fig. 11B) and modified (Fig. 11D) SEVAC samples. Some authors [34,37] relate the protein adsorption with surface topography rather than with surface chemistry and in this sense our results coincide with those reports since no notorious topographical changes were detected after the modification.

Cell adhesion mediated through extracellular adhesive proteins is involved in many intracellular signalling pathways that regulate most fundamental cell behaviours, including differentiation, proliferation and migration. We have compared the modified and untreated scaffolds by testing them in vitro with osteoblast-like cells SaOs-2. As it was expected and in agreement with the results which we obtained for protein adsorption no significant difference between both sets of samples was observed for short culture times (up to 7 days, Fig. 12). A close look at cells morphology reveals some differences at the initial time of culture. As mentioned before, some of the SaOs-2 cultured on untreated SEVA-C scaffolds for $24 \mathrm{~h}$ presented round shape (Fig. 12B) while cells onto modified samples were spread with extended
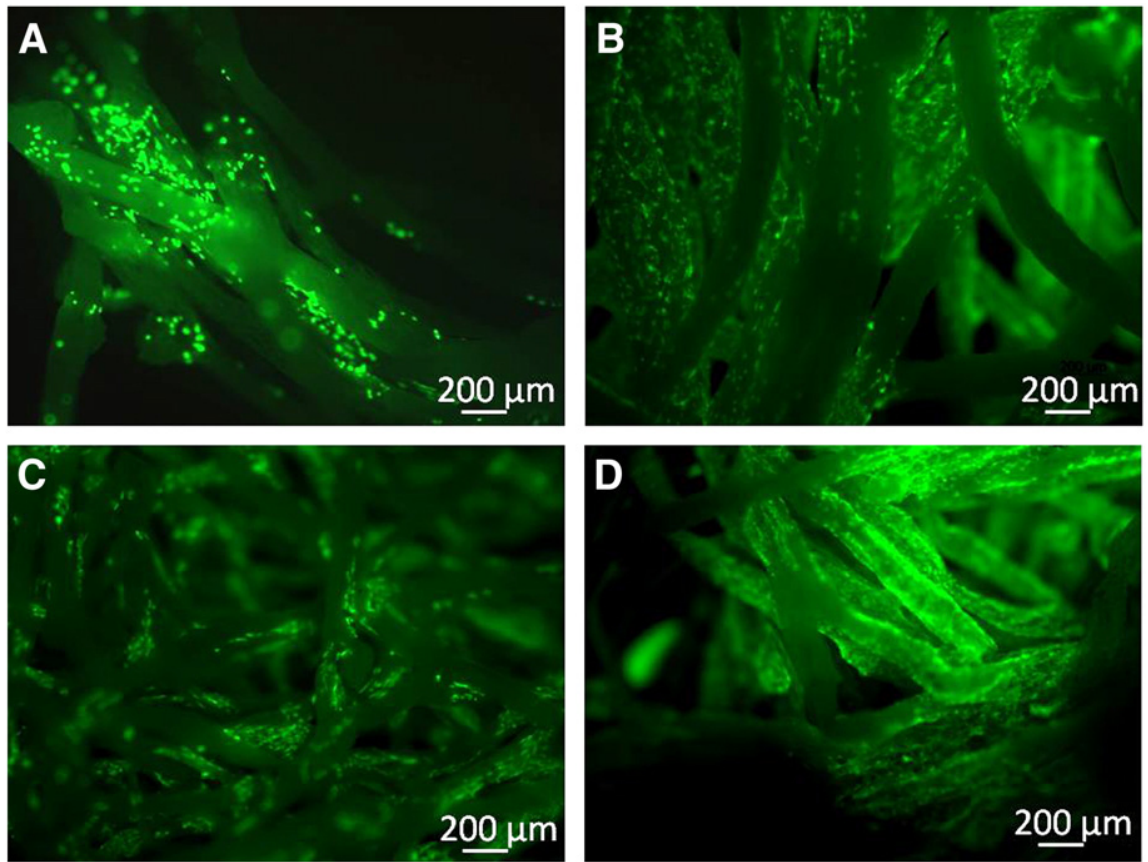

Fig. 13. Calcein-AM viability assay of SaOs-2 grown on untreated (A, B) and plasma-modified (C, D) scaffolds for 7 (left column) and 14 (right column) days. 
filopodias establishing contact between each other (Fig. 12C). However, after 3 days of culture and until the end of the studied period this difference in the morphology was not notorious and the cells were spread all along the fibers.

Calcein-AM assay showed another detail on cell distribution within the scaffolds: until the 7th day of culture cells grow and form random clusters on both sets of samples (Fig. 13A and C). After this time point, at 14 days of culture, they were homogeneously distributed (Fig. 13B and D) within the scaffolds. However, the difference between untreated and modified scaffolds is notorious: the detected fluorescence and hence the number of the viable cells increase for plasma treated scaffolds in comparison with the non-treated ones. These results were confirmed by the DNA quantification (Fig. 12A) where significantly higher DNA was detected for modified materials after 14 days of culture.

\section{Conclusions}

We have demonstrated that wet spinning technique is a viable alternative of the commonly used melt spinning for obtaining fine fibers from thermo-sensitive/degradable polymers. The obtained wet spun fibers from SEVA-C presented some features which distinguish them from the previously reported melt spun fibers: they are about 5 times thinner, much stiffer and they possess rough surface favouring their in vitro biological performance. The available high surface area raises the water uptake ability which turns the fibers and the scaffolds produced from them appropriate not only as supports for cell growth but also as a system for loading/releasing of bioactive compounds (e.g. growth factors). The morphology of the SEVA-C fiber mesh scaffolds reported herein for the first time is distinctive with favourable features that enhance osteoblasts adhesion and proliferation. The scaffolds also present an adequate pore size and a high interconnectivity allowing better transport inside the scaffold. Moreover, this morphology makes possible the application of physical surface modification techniques to the obtained 3D structures. We have shown in practice that plasma surface modification can be used to further improve the SEVA-C scaffolds. It must be noticed that the proposed method is only a proof of concept, confirming the versatility of the obtained structures and the ability to upgrade them but the modification strategies are not limited to this method. Other alternatives such as plasma functionalisation using gases different that Ar or plasma grafting of bioactive entities can be also used for functionalisation targeting specific applications.

\section{Acknowledgments}

This work was partially supported by the European NoE EXPERTISSUES (NMP3-CT-2004-500283), EU Marie Curie Actions, Alea Jacta Est (MEST-CT-2004-008104) and FCT project PTDC/CTM/67560/2006. The authors would also like to acknowledge Sofia G. Caridade and Dr Marina I. Santos for their excellent technical assistance with the DMA and confocal microscopy, respectively.

\section{References}

[1] D. Eagles, D. Lesnoy, S. Barlow, Textile Research Journal 66 (1996) 277.

[2] I.S. Arvanitoyannis, Journal of Macromolecular Science-Reviews in Macromolecular Chemistry and Physics C39 (1999) 205.

[3] L. Averous, Journal of Macromolecular Science-Polymer Reviews C44 (2004) 231.

[4] N.A. Dung, N.D. Huyen, N.D. Hang, T.T. Canh, Radiation Physics and Chemistry 46 (1995) 1037.

[5] R.L. Reis, A.M. Cunha, Journal of Materials Science-Materials in Medicine 6 (1995) 786.

[6] E.-J. Lee, D.-K. Kweon, B.-K. Koh, S.-T. Lim, Journal of Applied Polymer Science 92 (2004) 861

[7] E. Chiellini, A. Corti, S. D'Antone, R. Solaro, Progress in Polymer Science 28 (2003) 963.

[8] C. Bastioli, Polymer Degradation and Stability 59 (1998) 263.

[9] J.F. Mano, G.A. Silva, H.S. Azevedo, P.B. Malafaya, R.A. Sousa, S.S. Silva, L.F. Boesel, J.M. Oliveira, T.C. Santos, A.P. Marques, N.M. Neves, R.L. Reis, Journal of the Royal Society Interface 4 (2007) 999.

[10] E. Chiellini, P. Cinelli, V.I. Ilieva, S.H. Imam, J.W. Lawton, Journal of Cellular Plastics 45 (2009) 17.

[11] C. Bastioli, V. Bellotti, L. Del Guidice, R. Lombi, Polymer Compositions for the Production of Articles of Biodegradable Plastics Material and Methods for their Preparation, NOVAMONT SPA, 1990.

[12] C. Bastioli, Starch-Starke 53 (2001) 351.

[13] R.L. Reis, A.M. Cunha, P.S. Allan, M.J. Bevis, Polymers for Advanced Technologies 7 (1996) 784

[14] R.A. Sousa, R. Reis, A. Cunha, M.J. Bevis, Composites Science and Technology 63 (2003) 389.

[15] R.L. Reis, S.C. Mendes, A.M. Cunha, M.J. Bevis, Polymer International 43 (1997) 347.

[16] H.S. Azevedo, F.M. Gama, R.L. Reis, Biomacromolecules 4 (2003) 1703.

[17] C.M. Vaz, R.L. Reis, A.M. Cunha, Materials Research Innovations 4 (2001) 375

[18] S.C. Mendes, R.L. Reis, Y.P. Bovell, A.M. Cunha, C.A. van Blitterswijk, J.D. de Bruijn, Biomaterials 22 (2001) 2057.

[19] M.E. Gomes, R.L. Reis, A.M. Cunha, C.A. Blitterswijk, J.D. de Bruijn, Biomaterials 22 (2001) 1911.

[20] M.E. Gomes, A.S. Ribeiro, P.B. Malafaya, R.L. Reis, A.M. Cunha, Biomaterials 22 (2001) 883.

[21] M.E. Gomes, J.S. Godinho, D. Tchalamov, A.M. Cunha, R.L. Reis, Materials Science \& Engineering C-Biomimetic and Supramolecular Systems 20 (2002) 19.

[22] M.P. Pavlov, J.F. Mano, N.M. Neves, R.L. Reis, Macromolecular Bioscience 4 (2004) 776.

[23] I. Pashkuleva, H.S. Azevedo, R. Reis, Macromolecular Bioscience 8 (2008) 210.

[24] S. Simmons, E.L. Thomas, Journal of Applied Polymer Science 58 (1995) 2259.

[25] Y. Luo, G. Engelmayr, D.T. Auguste, Three-dimensional Scaffolds, in: R. Lanza, R. Langer, J.P. Vacanti (Eds.), Principles of Tissue Engineering. Elsevier Inc., 2007, p. 359.

[26] Y. Kuboki, Q.M. Jin, H. Takita, Journal of Bone and Joint Surgery-American Volume 83A (2001) S105.

[27] J.F. Mano, D. Koniarova, R.L. Reis, Journal of Materials Science-Materials in Medicine 14 (2003) 127

[28] P.J. Stenhouse, J.A. Ratto, N.S. Schneider, Journal of Applied Polymer Science 64 (1997) 2613.

[29] C.R. Kothapalli, M.T. Shaw, M. Wei, Acta Biomaterialia 1 (2005) 653.

[30] A.P. Marques, R.L. Reis, J.A. Hunt, Biomaterials 23 (2002) 1471

[31] A.J. Salgado, O.P. Coutinho, R.L. Reis, Tissue Engineering 10 (2004) 465.

[32] A.J. Salgado, O.P. Coutinho, R.L. Reis, J.E. Davies, Journal of Biomedical Materials Research Part A 80A (2007) 983.

[33] C.M. Alves, Y. Yang, D.L. Carnesc, J.L. Ong, V.L. Sylvia, D.D. Dean, C.M. Agrawal, R.L. Reis, Biomaterials 28 (2007) 307.

[34] M.I. Santos, I. Pashkuleva, C.M. Alves, M.E. Gomes, S. Fuchs, R.E. Unger, R.L. Reis, J. Kirkpatrick, Journal of Materials Chemistry 19 (2009) 4091.

[35] C.M. Alves, R.L. Reis, J.A. Hunt, Journal of Materials Science: Materials in Medicine 14 (2003) 157.

[36] C.C. Barrias, M.C.L. Martins, G. Almeida-Porada, M.A. Barbosa, P.L. Granja, Biomaterials 30 (2009) 307.

[37] T.J. Webster, C. Ergun, R.H. Doremus, R.W. Siegel, R. Bizios, Journal of Biomedical Material Research 51 (2000) 475. 